\title{
Comparison Between the Prognostic Value of Left Ventricular Function and Myocardial Perfusion Reserve in Patients with Ischemic Heart Disease
}

René A. Tio*1, Ali Dabeshlim*2, Hans-Marc J. Siebelink ${ }^{3}$, Johan de Sutter ${ }^{4}$, Hans L. Hillege ${ }^{5}$, Clark J. Zeebregts ${ }^{6}$, Rudi A.J.O. Dierckx², Dirk J. van Veldhuisen ${ }^{1}$, Felix Zijlstra ${ }^{1}$, and Riemer H.J.A. Slart ${ }^{2}$

${ }^{1}$ Department of Cardiology, University Medical Center Groningen, Groningen, The Netherlands; ${ }^{2}$ Department of Nuclear Medicine and Molecular Imaging, University Medical Center Groningen, Groningen, The Netherlands; ${ }^{3}$ Department of Cardiology, Leiden University Medical Center, Leiden, The Netherlands; ${ }^{4}$ Department of Cardiology, AZ Maria Middelares, Ghent, Belgium;

${ }^{5}$ Department of Epidemiology, University Medical Center Groningen, Groningen, The Netherlands; and ${ }^{6}$ Department of Surgery, Division of Vascular Surgery, University Medical Center Groningen, Groningen, The Netherlands

The purpose of this study was to compare the prognostic value of left ventricular ejection fraction (LVEF) and myocardial perfusion reserve (MPR) assessed with PET in patients with ischemic heart disease (IHD). Myocardial perfusion is the main determinant of left ventricular function in patients with IHD. The prognostic value of LVEF has been widely established. In addition, MPR determines survival in patients with hypertrophic and dilated cardiomyopathies. In the present study, we evaluated whether MPR also determines survival in patients with IHD. Methods: Between 1995 and 2003, 480 consecutive patients with chronic IHD underwent dipyridamole stress and rest ${ }^{13} \mathrm{~N}$-ammonia PET to determine MPR. Additionally, ${ }^{18}$ F-FDG PET was performed for viability (mismatching defects), infarction (matching defects), and left ventricular function assessment. Patients were followed for all causes of mortality and major cardiovascular events. Results: In 463 of the 480 patients, valid MPR could be measured (368 men; mean age, $66 \pm 11 \mathrm{y}$; LVEF, 35\% $\pm 15 \%$ ). One hundred nineteen patients underwent a PET-driven revascularization (67 through percutaneous coronary intervention and 52 through coronary artery bypass grafting). The remaining 344 patients were the subject of this study. The overall MPR was $1.71 \pm 0.50$ (intertertile boundaries, 1.49 and 1.84). After adjustment for age and sex, MPR was associated with a hazard ratio for cardiac death of 4.11 (95\% confidence interval, 2.985.67) per SD decrease, whereas the risk for LVEF was 2.76 (2.00-3.82) per SD decrease. Conclusion: Patients with IHD with a low MPR are at high risk of cardiac death. MPR is a more sensitive predictor for cardiac death than is LVEF.

Received May 16, 2008; revision accepted Jul. 25, 2008.

For correspondence or reprints contact: Riemer H.J.A. Slart, Department of Nuclear Medicine and Molecular Imaging, University Medical Center Groningen, University of Groningen, Hanzeplein 1, P.O. Box 30001, 9700 RB Groningen, The Netherlands.

E-mail: r.h.j.a.slart@ngmb.umcg.nl

${ }^{*}$ Contributed equally to this work.

COPYRIGHT ๑ 2009 by the Society of Nuclear Medicine, Inc.
Key Words: positron emission tomography; myocardial blood flow quantification; coronary artery disease; endothelial function; prognosis; LV function

J Nucl Med 2009; 50:214-219

DOI: 10.2967/jnumed.108.054395

I schemic heart disease (IHD) is a progressive disease eventually leading to loss of ventricular function and cardiac death (1). Patients with one or more previous myocardial infarctions and one or more coronary interventions often pose a difficult therapeutic dilemma. The question arises of whether to treat only medically or to aim also (again) at an intervention. Careful analysis of myocardial perfusion in combination with viability and function can guide patienttailored therapeutic strategies (2). PET using ${ }^{18} \mathrm{~F}-\mathrm{FDG}$ combined with the flow tracer ${ }^{13} \mathrm{~N}$-ammonia is an accurate, noninvasive diagnostic technique to assess myocardial viability and ischemia in patients with chronic $\operatorname{IHD}(3,4)$ It has been shown that not only the extent of PET-based viable myocardium but also the extent of infarcted myocardium is an important predictor of left ventricular function recovery after revascularization (5). Most PET studies for analysis of patient survival after treatment have been based on semiquantitative scoring of myocardial perfusion and ${ }^{18} \mathrm{~F}-\mathrm{FDG}$ distribution. Dynamic imaging with PET allows the quantitative assessment of myocardial tracer kinetics and, hence, the measurement of physiologic processes such as myocardial blood flow, using the model of Hutchins et al. (6). In addition, and in contrast to SPECT, PET enables absolute measurements of myocardial blood flow, permitting the assessment of coronary perfusion reserve.

In previous studies, the prognostic value of myocardial perfusion reserve (MPR) was established in patients without coronary artery disease (7), in patients with hypertrophic 
cardiomyopathy $(8,9)$, and in patients with idiopathic left ventricular dysfunction $(10,11)$. Whether this prognostic value holds true for patients with epicardial IHD is unknown. Therefore, we investigated whether, in comparison with left ventricular ejection fraction (LVEF), MPR assessed with PET using absolute myocardial blood flow quantification and perfusion reserve can predict survival in a large group of patients with IHD.

\section{MATERIALS AND METHODS}

\section{Patients and Study Design}

This study prospectively included, with retrospective analysis, 480 subjects with advanced IHD who underwent rest ${ }^{13} \mathrm{~N}$-ammonia, dipyridamole stress ${ }^{13} \mathrm{~N}$-ammonia, and gated ${ }^{18} \mathrm{~F}$-FDG PET, between 1995 and 2003 at the PET center of the University Medical Center Groningen, for evaluation of stress-induced ischemia and myocardial viability. Patient data were collected from the hospital information system (Table 1). PET-driven intervention was defined as any cardiac (surgical or percutaneous) procedure performed within the first 6 mo after the PET study date. If no intervention was performed, the patients were considered to be only medically treated.

\section{Endpoints}

All causes of mortality were assessed. Cardiac death was defined as sudden death, death after the onset of symptoms suggestive of cardiac ischemia, or death due to heart failure. Cardiac events included cardiac death, myocardial infarction, and nonPET-driven revascularization. Myocardial infarction was defined as an increase in cardiac enzymes $(>2 \times$ the upper limit of normal), new pathologic $\mathrm{Q}$ waves on the electrocardiogram, or both. A major adverse cardiac event was defined as cardiac death, myocardial infarction, percutaneous coronary intervention (PCI), coronary artery bypass grafting (CABG), or hospitalization for an acute coronary syndrome or heart failure.

\section{PET}

The patients underwent dynamic rest ${ }^{13} \mathrm{~N}$-ammonia, dipyridamole stress ${ }^{13} \mathrm{~N}$-ammonia, and gated ${ }^{18} \mathrm{~F}$-FDG PET using a 1-d protocol, as described previously (12). Briefly, PET studies were performed after the patients had discontinued vasoactive medica-

TABLE 1. Patient Characteristics for Each Tertile of MPR

\begin{tabular}{|c|c|c|c|c|c|}
\hline Characteristic & $\begin{array}{l}\text { MPR, first } \\
\text { tertile }(<1.49) \\
(n=114)\end{array}$ & $\begin{array}{l}\text { MPR, second } \\
\text { tertile }(1.49-1.84) \\
\quad(n=116)\end{array}$ & $\begin{array}{l}\text { MPR, third } \\
\text { tertile }(>1.84) \\
(n=114)\end{array}$ & $\begin{array}{c}\text { Total } \\
(n=344)\end{array}$ & $P$ \\
\hline Age (y) & $69 \pm 11$ & $68 \pm 10$ & $62 \pm 11$ & $66 \pm 11$ & $<0.001$ \\
\hline $\operatorname{Sex}(\mathrm{F} / \mathrm{M})(n)$ & $22 / 92$ & $26 / 90$ & $25 / 89$ & $73 / 271$ & 0.628 \\
\hline Hypercholesterolemia (\%) & 54 & 53 & 64 & 57 & 0.141 \\
\hline Smoking (\%) & 31 & 28 & 38 & 33 & 0.323 \\
\hline Diabetes mellitus (\%) & 12 & 17 & 10 & 13 & 0.539 \\
\hline Hypertension (\%) & 28 & 29 & 29 & 29 & 0.884 \\
\hline Family history (\%) & 32 & 40 & 54 & 42 & 0.001 \\
\hline Previous myocardial infarction (\%) & 82 & 71 & 61 & 71 & $<0.001$ \\
\hline Previous CABG (\%) & 29 & 31 & 26 & 29 & 0.661 \\
\hline Previous $\mathrm{PCl}(n)$ & 44 & 42 & 48 & 45 & 0.506 \\
\hline LVEF (\%) & $30 \pm 14$ & $37 \pm 13$ & $41 \pm 17$ & $36 \pm 15$ & $<0.001$ \\
\hline Typical anginal complaints (\%) & 77 & 85 & 81 & 81 & 0.747 \\
\hline Left ventricular end-diastolic volume $(\mathrm{mL})$ & $172 \pm 91$ & $124 \pm 62$ & $124 \pm 81$ & $140 \pm 82$ & $<0.001$ \\
\hline Aspirin (\%) & 54 & 68 & 68 & 63 & 0.040 \\
\hline Coumarin (\%) & 44 & 29 & 25 & 33 & 0.003 \\
\hline Statin (\%) & 52 & 48 & 61 & 54 & 0.185 \\
\hline Diuretic (\%) & 47 & 31 & 24 & 34 & $<0.001$ \\
\hline Digoxin (\%) & 22 & 11 & 8 & 14 & 0.002 \\
\hline$\beta$-blocker (\%) & 64 & 70 & 60 & 65 & 0.489 \\
\hline $\begin{array}{l}\text { Angiotensin-converting } \\
\text { enzyme-inhibitor/ARB (\%) }\end{array}$ & 58 & 40 & 42 & 47 & 0.017 \\
\hline Matching defect (\% left ventricle) & $30 \pm 15$ & $27 \pm 15$ & $25 \pm 17$ & $27 \pm 16$ & 0.012 \\
\hline $\begin{array}{l}\text { Location of matching defects: } \\
\text { LAD/LCX/RCA area (\%) }\end{array}$ & $29 / 33 / 44$ & $34 / 30 / 55$ & $32 / 18 / 51$ & $31 / 27 / 50$ & $0.669 / 0.007 / 0.290$ \\
\hline Mismatching defect (\% left ventricle) & $11 \pm 10$ & $8 \pm 10$ & $7 \pm 9$ & $9 \pm 10$ & 0.011 \\
\hline $\begin{array}{l}\text { Location of mismatching defects: } \\
\text { LAD/LCX/RCA area (\%) }\end{array}$ & $27 / 37 / 33$ & $26 / 24 / 28$ & $14 / 33 / 22$ & $22 / 31 / 28$ & $0.017 / 0.393 / 0.055$ \\
\hline Normal area of left ventricle (\%) & $59 \pm 19$ & $65 \pm 19$ & $68 \pm 21$ & $64 \pm 20$ & 0.001 \\
\hline Myocardial perfusion at rest $(\mathrm{mL} / \mathrm{min} / 100 \mathrm{~g})$ & $96 \pm 23$ & $85 \pm 22$ & $80 \pm 21$ & $87 \pm 23$ & $<0.001$ \\
\hline $\begin{array}{l}\text { Myocardial perfusion during } \\
\text { stress }(\mathrm{mL} / \mathrm{min} / 100 \mathrm{~g})\end{array}$ & $117 \pm 34$ & $139 \pm 36$ & $177 \pm 45$ & $145 \pm 46$ & $<0.001$ \\
\hline $\begin{array}{l}\mathrm{ARB}=\text { angiotensin type I receptor blocker; } \\
\text { right coronary artery. } \\
\text { Categoric variables are show as percentag }\end{array}$ & $\begin{array}{l}D=\text { left anter } \\
\text { or as number }\end{array}$ & $\begin{array}{l}\text { descending coro } \\
\text { continuous variabl }\end{array}$ & / artery; LCX & rcumflex & onary artery; $\mathrm{RCA}=$ \\
\hline
\end{tabular}


tion for 5 plasma half-lives and had refrained from caffeinated beverages for a minimum of $12 \mathrm{~h}$. Imaging was performed with the patient supine and used an ECAT 951 positron camera (Siemens CTI). Thirty-one planes were measured simultaneously over a length of $10.8 \mathrm{~cm}$. The measured resolution of the system was $6 \mathrm{~mm}$ in full width at half maximum. Data were automatically corrected for accidental coincidence and dead time. Patients were positioned with the help of a rectilinear scan. Photon attenuation was measured using a retractable external ring source filled with ${ }^{68} \mathrm{Ge} /{ }^{68} \mathrm{Ga}$. Perfusion imaging was performed after dipyridamole had been infused $(0.56 \mathrm{mg} / \mathrm{kg}$ in $4 \mathrm{~min})$. Imaging was started by injecting $400 \mathrm{MBq}$ of ${ }^{13} \mathrm{~N}$-ammonia $6 \mathrm{~min}$ after the start of dipyridamole infusion and continued for $15 \mathrm{~min}$.

To stimulate ${ }^{18} \mathrm{~F}$-FDG uptake, patients were given $75 \mathrm{~g}$ of glucose orally just before scanning or were given $500 \mathrm{mg}$ of acipimox (Nedios; Byk Pharmaceuticals) orally $90 \mathrm{~min}$ before scanning to lower circulating free fatty acids (13). To prevent side effects of acipimox (e.g., skin rash), $250 \mathrm{mg}$ of aspirin were administered orally $5 \mathrm{~min}$ before acipimox. In diabetic patients, ${ }^{18} \mathrm{~F}-\mathrm{FDG}$ imaging was done with hyperinsulinemic euglycemic glucose clamping (14). After the ${ }^{13} \mathrm{~N}$-ammonia data had been acquired, $200 \mathrm{MBq}$ of ${ }^{18} \mathrm{~F}-\mathrm{FDG}$ were injected intravenously, followed by a PET dynamic acquisition. The total ${ }^{18}$ F-FDG PET acquisition time was $40 \mathrm{~min}$, with the last $20 \mathrm{~min}$ acquired in gated mode with 16 frames per cardiac cycle. The length of each gate was based on the current R-R interval. The R-R interval was allowed to vary by $\pm 10 \%$. Data were corrected for attenuation using the transmission scan and were reconstructed using filtered backprojection (Hann filter, 0.5 pixels/cycle).

\section{Kinetic Models and Data Analysis}

From the PET data, dynamic parametric polar maps were constructed (12). PET perfusion data at rest were corrected for rate-pressure product. Myocardial blood flow data were corrected for partial-volume effect and spillover and quantified by the model of Hutchins et al. (6). Briefly, myocardial and blood time-activity curves derived from regions of interest over the heart and ventricular chamber are fitted using a 3-compartment model for ${ }^{13} \mathrm{~N}$-ammonia, yielding rate constants for tracer uptake and retention. Perfusion flow reserve (dipyridamole-to-rest ratio) was calculated by dividing the dipyridamole ${ }^{13} \mathrm{~N}$-ammonia stress study by the ${ }^{13} \mathrm{~N}$-ammonia rest study.

Data analysis of ${ }^{18} \mathrm{~F}-\mathrm{FDG}$ was performed with PATLAK analysis (15).

Mismatch was quantified by first normalizing the ${ }^{18} \mathrm{~F}-\mathrm{FDG}$ uptake polar map and the dipyridamole blood flow polar map to their means. Then, a difference polar map was created by subtracting the normalized dipyridamole blood flow polar map from the normalized ${ }^{18}$ F-FDG uptake polar map. Mismatch was calculated as the percentage myocardium above the $95 \%$ confidence interval of the normal database, and results were expressed as percentage of the total myocardium. Similarly, matching areas were quantified by constructing a product polar map; the normalized dipyridamole blood flow polar map was multiplied by the normalized ${ }^{18} \mathrm{~F}$-FDG uptake polar map. Match was defined as the percentage myocardium below the $95 \%$ confidence interval. The extent of mismatching areas (viable myocardium) and matching areas (nonviable myocardium) was calculated from these data as previously described (12).

The last frames (20-min acquisition time) of the dynamic gated ${ }^{18}$ F-FDG PET studies were summed and transformed into static studies and used for further data analysis with the help of the quantitative gated SPECT program (15). Based on the gated ${ }^{18}$ F-FDG images, left ventricular end-systolic and end-diastolic volumes, as well as LVEF, were computed.

\section{Statistical Analysis}

Descriptive results are expressed as mean \pm SD. Categoric measures are presented as frequencies with percentages. Crude data were compared across tertiles of MPR, defined as perfusion during dipyridamole divided by resting perfusion, with the $\chi^{2}$ test for trend (dichotomous variables) and generalized linear models (continuous variables). The significance of MPR, controlled for important risk modifiers as presented in Table $1(P<0.20)$, was examined with multivariable Cox proportional hazards regression analyses by using fractional polynomials (16). Results are summarized by hazard (risk) ratios with confidence intervals based on robust SE estimates. To assess the prognostic value of LVEF and MPR adjusted for age and sex, Harrell's C-statistic was computed (comparable to the area under the receiver-operating-characteristic curve). Model fit was assessed with Bayesian information criterion statistics, which are goodness-of-fit measures adjusted by degrees of freedom and sample size. Smaller Bayesian information criterion values indicate that the model fits better. A difference of 10 points or more between a given model and the other model is strong evidence for a significantly better goodness of fit. The significance level was set at 0.05 . Observations with missing values for contributing variables in the multivariate model were excluded. The statistical analysis was performed with SPSS (SPSS Inc.), version 9.1, and STATA statistical software, release 10.0 (StataCorp LP).

\section{RESULTS}

\section{Patient Characteristics}

Between 1995 and 2003, 480 patients underwent a ${ }^{13} \mathrm{~N}-$ ammonia rest, a dipyridamole stress, and a gated ${ }^{18} \mathrm{~F}-\mathrm{FDG}$ PET scan. In 17 patients, gating was not possible because of heart rate irregularities occurring during the scan. In 463 patients, valid MPR could be measured. One hundred nineteen patients (368 men; mean age, $66 \pm 11 \mathrm{y}$; LVEF, $35 \% \pm$ $15 \%$ ) underwent a PET-driven revascularization (67 through PCI and 52 through CABG). Patients with a PET-driven intervention were comparable to the study group with respect to age $(66 \pm 11 \mathrm{y}$ vs. $68 \pm 10 \mathrm{y})$, sex $(22 \%$ female vs. $18 \%$ female), risk factors, previous myocardial infarction $(71 \%$ vs. $78 \%$ ), and previous PCIs (45\% vs. $36 \%$ ) but had significantly more previous CABGs $(28 \%$ vs. $15 \%, P=0.028)$ and a higher LVEF $(36 \pm 16$ vs. $32 \pm 14, P=0.007)$. The remaining 344 patients were the subject of this study. The baseline characteristics of these 344 patients are shown in Table 1: $14 \%$ of the patients were in NYHA class I, $49 \%$ in class II, $31 \%$ in class III, and $5 \%$ in class IV. Overall, the MPR was $1.71 \pm 0.50$ (intertertile boundaries, 1.49 and 1.84). Areas of mismatch were found in 91 patients $(27 \%)$, areas of matching defects in $267(78 \%)$, both mismatching and matching defects in $80(23 \%)$, and no defects at all in 66 (19\%). Mean percentage match in the study group was $27 \% \pm 16 \%$, mean percentage mismatch was $9 \% \pm 10 \%$, and mean LVEF was $36 \% \pm 15 \%$. Coronary artery disease was present in the left anterior descending artery in $66 \%$ of 
patients, in the right coronary artery in $43 \%$, and in the circumflex coronary artery in $30 \%$.

\section{Outcome Event}

The median follow-up among survivors was 85 mo (range, $1-138 \mathrm{mo}$ ). Among the 344 patients in this study, there were a total of 85 deaths $(25 \%)$, of which 60 (17\%) were cardiac deaths. Twenty-five patients $(7 \%)$ experienced a nonfatal myocardial infarction. A total of 71 patients $(21 \%)$ underwent a PCI and 27 a CABG $(8 \%)$ during follow-up.

\section{Hazard Ratio of MPR}

Table 2 summarizes the results of the Cox regression analysis for cardiac death. In the univariate analysis, the parameters significantly associated with cardiac death were MPR; family history; previous myocardial infarction; LVEF; left ventricular end-diastolic volume; the use of aspirin, diuretics, or digoxin; and matching. After controlling for age and sex, the following parameters were associated with cardiac death: MPR, family history, previous myocardial infarction, LVEF, left ventricular end-diastolic volume, aspirin, diuretics, and digoxin. MPR was associated with a hazard ratio for cardiac death of 4.11 (95\% confidence interval, 2.98-5.67) per SD decrease, whereas the risk for LVEF was 2.76 (2.00-3.82) per SD decrease. Interestingly, the prognostic value of MPR was independent of the extent of matching and mismatching defects. Survival data for each MPR tertile are shown in Figure 1. The hazard function of MPR, when compared with LVEF, was steeper in a prognostic model adjusted for age and sex, resulting in improved C-statistics and Bayesian information criterion statistics (0.83, 605 vs. 0.77, 620) (Fig. 2). Finally, in a secondary mutually adjusted multivariate analysis of MPR; family history; previous myocardial infarction; LVEF; left ventricular end-diastolic volume; the use of aspirin, diuretics, or digoxin; and percentage matching defects, the parameters that remained statistically significant in the model were MPR, LVEF, and the use of diuretics (hazard ratios of 4.08
[2.50-6.65, $P<0.001], 1.91[1.10-3.31, P=0.021]$, and 2.19 [1.07-4.97, $P=0.033]$, respectively).

\section{Hazard Ratio for Major Adverse Cardiac Event}

After univariate analysis of baseline demographics (including PET parameters) MPR had a hazard ratio of 1.60 (1.31-1.94, $P<0.001)$ for major adverse cardiac events. Finally, in a secondary mutually adjusted multivariate analysis, MPR remained statistically significant in the model (hazard ratio, $1.44[1.14-1.84, P=0.003]$ ).

Interestingly, the prognostic value of MPR for cardiac death and major adverse cardiac events was independent of whether patients received a PET-driven medical strategy or a revascularization strategy and of the extent of matching or mismatching defects.

\section{DISCUSSION}

It has been shown that MPR is of prognostic value in patients without coronary artery disease (7) and in patients with hypertrophic or idiopathic cardiomyopathy (8-10). The present study shows comparable results in patients with IHD. Interestingly, these findings were independent of the extent of myocardial ischemia or infarction and superior to the prognostic value of LVEF. Further, a small decrease in MPR was associated with a large increment in mortality rate and showed an improved fit when compared with LVEF.

Although the prognostic value of MPR in subgroups of patients without obstructive coronary artery disease has been established, the prognostic value of PET in patients with obstructive coronary artery disease has been evaluated only with respect to areas of matching or mismatching defects (16). In previous studies, an association between the presence or extent of ischemic myocardial area and survival has been described (16-18). In 2 of these studies, the presence of mismatching defects (without absolute quantification) has been described in relation to prognosis $(17,18)$. In contrast to the previous studies, we did not find an association between the presence or extent of mismatching defects and progno-

TABLE 2. Univariate and Multivariate Cox Proportional Hazard Regression Analysis of Cardiac Death According to MPR

\begin{tabular}{|c|c|c|c|c|}
\hline \multirow[b]{2}{*}{ Variable } & \multicolumn{2}{|c|}{ Univariate analysis } & \multicolumn{2}{|c|}{ Age- and sex-adjusted analysis } \\
\hline & Hazard ratio & $P$ & Hazard ratio & $P$ \\
\hline MPR (per SD) & $4.18(3.05-5.74)$ & $<0.001$ & $4.11(2.98-5.67)$ & $<0.001$ \\
\hline Family history (yes) & $0.56(0.32-0.98)$ & 0.043 & $0.68(0.38-1.21)$ & 0.191 \\
\hline Previous myocardial infarction (yes) & $6.06(2.20-16.71)$ & 0.001 & $5.21(1.87-14.54)$ & 0.002 \\
\hline LVEF (per SD) & $2.79(2.02-3.85)$ & $<0.001$ & $2.76(2.00-3.82)$ & $<0.001$ \\
\hline LVEDV (per $10 \mathrm{~mL}$ ) & $1.05(1.03-1.08)$ & $<0.001$ & $1.06(1.04-1.08)$ & $<0.001$ \\
\hline Aspirin (yes) & $0.41(0.24-0.67)$ & 0.001 & $0.40(0.24-0.68)$ & $<0.001$ \\
\hline Diuretics (yes) & $5.58(3.17-9.81)$ & $<0.001$ & $5.58(3.15-9.91)$ & $<0.001$ \\
\hline Digoxin (yes) & $4.97(2.94-8.39)$ & $<0.001$ & $4.94(2.90-8.40)$ & $<0.001$ \\
\hline Matching (per 10\%) & $1.40(1.20-1.64)$ & $<0.001$ & $1.41(1.20-1.66)$ & $<0.001$ \\
\hline Mismatching (per 10\%) & $1.20(0.95-1.51)$ & 0.134 & $1.16(0.91-1.50)$ & 0.226 \\
\hline
\end{tabular}




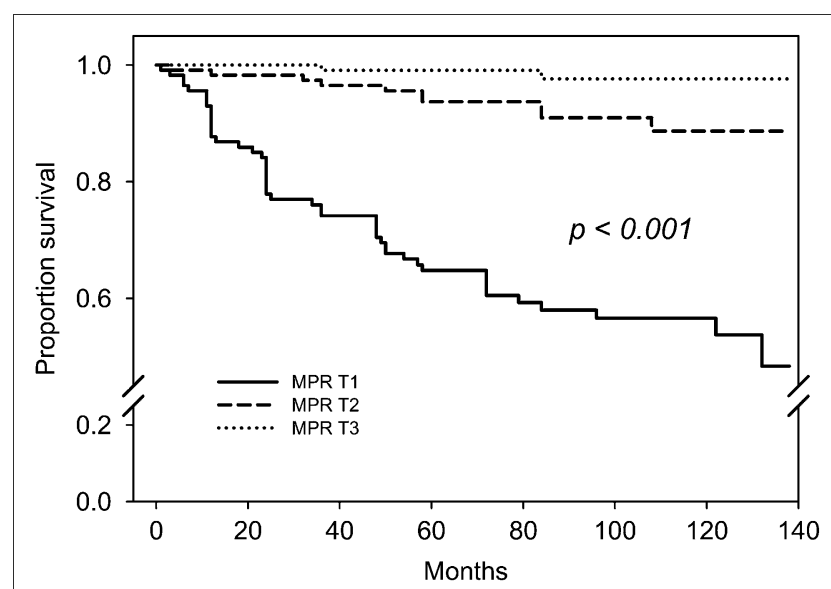

MPR T1 114 (16) $96 \quad$ (13) 80 (10) 64 (5) $47 \quad$ (2) 38 (0) $22 \quad$ (1) 13 MPR T2 116 (2) 113 (2) 107 (3) 101 (0) 72 (2) 51 (1) $31 \quad$ (0) 14

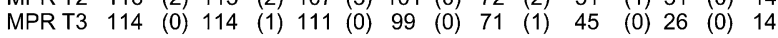

FIGURE 1. Cardiac death-free survival curves for median values of MPR tertiles are shown. Median MPR values of each tertile (intertertile boundaries, 1.49 and 1.84) were taken to illustrate differences between these groups. Numbers indicate patients at risk and number of events per tertile $(T)$.

sis. The percentage of patients with a previous myocardial infarction $(52 \%-87 \%)$ was comparable to that in our study group $(73 \%)$. An explanation may be that in our patient group a larger proportion of patients had undergone a previous coronary intervention: $26 \% \mathrm{CABG}$ and $43 \% \mathrm{PCI}$ in our group, versus $6 \%-10 \%$ and $8 \%-9 \%$, respectively, in the study of Desideri et al. (16) or even as low as $3 \%$ in the study of Wiggers et al. (18). The higher percentage of patients with a previous revascularization may have resulted in much smaller ischemic areas $(10 \% \pm 10 \%)$ in our study than in the previous study by Desideri et al. $(36 \%-58 \%)$. Previous SPECT perfusion imaging studies are also in line with our MPR data: increasing perfusion abnormalities were associated with worsening prognosis (19). However, SPECT is not able to quantify absolute perfusion and may underdiagnose ischemia in patients with severe 3-vessel or left main coronary disease.

Our group is comparable to a previously studied group of ischemic heart failure patients with respect to the high rate of prior interventions (20). In that study, patients with ejection fractions of $23 \% \pm 7 \%$ were evaluated. It appeared that in approximately $12 \%$ of these patients, viable myocardial segments (ischemia or hibernation) were present. These patients underwent a revascularization procedure if possible. Interestingly, in the lowest-tertile MPR group of our study, survival rates were comparable to those of the intervention group in the previous study, despite the fact that patients in our group had higher LVEFs. In contrast, patients in the higher MPR tertiles had better survival rates. Our study group consisted of a mixed population with regard to LVEF and was comparable to patients seen in clinical practice.

We did not analyze regional perfusion defects, but the fact that global perfusion reserve has such an impact on prognosis
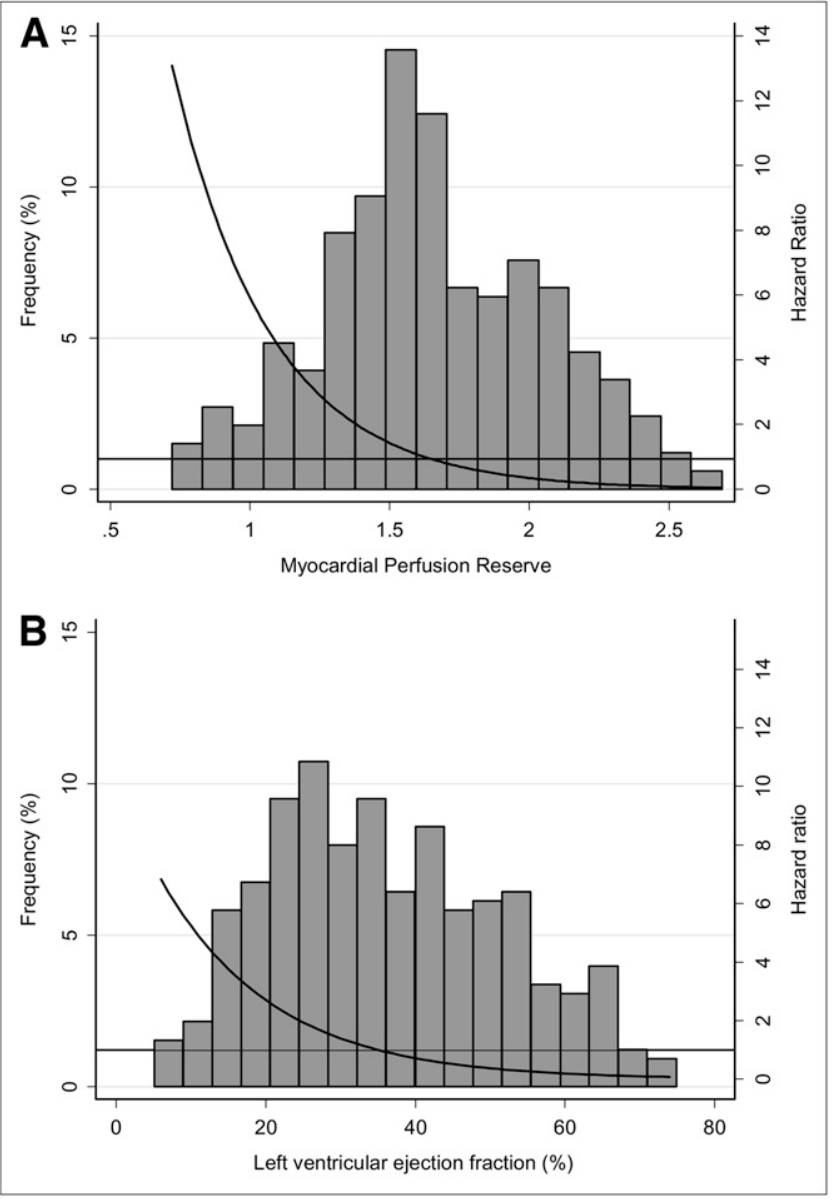

FIGURE 2. Hazard ratio curves and percentage distribution for MPR $(A)$ and for LVEF (B).

may relate to vascular dysfunction that has extended beyond the areas of stenotic coronary arteries. MPR depends mainly on the dilatory capacity of the prearteriolar sphincters in the microvasculature. Microvascular function is determined by metabolic need, structural changes, neurohumoral factors, autonomic innervation, extravascular resistance, and endothelial function. Among these factors, the endothelial component has been investigated the most extensively. Microvascular dysfunction can be found in patients without myocardial or obstructive coronary artery disease and is most often related to conventional risk factors such as smoking (21), hyperlipidemia (22,23), and diabetes (23). In addition, microvascular dysfunction can be the cause of angina pectoris in the absence of epicardial coronary disease, or the socalled syndrome-X (24). Myocardial perfusion abnormalities may influence myocardial contractility, but left ventricular dysfunction can also be mirrored by myocardial perfusion abnormalities (25).

In dilated cardiomyopathy, we have previously shown that despite the absence of IHD, regions with a lower MPR are present (11). The present study further expands these perfusion reserve data to patients with coronary artery disease. MPR can be considered a reflection of global ischemia and 
hence of the severity of coronary artery disease and left ventricular dysfunction. On the one hand, ischemia may lead to left ventricular dysfunction, but on the other hand, left ventricular dysfunction may cause abnormalities in the microvasculature (25).

Our study had some limitations. Because of the long follow-up period, a large proportion of patients underwent PET in the early 1990s. As a consequence, many patients with heart failure were still on digoxin therapy. However, the results did not differ between these patients and patients on angiotensin-converting-enzyme inhibitors. The metabolic state of the patients was not assessed at the moment of PET; however, neither fasting glucose levels nor diabetes mellitus influenced our multivariate model. The implication is that MPR is a robust determinant of prognosis, independent of the metabolic state of the patients. Furthermore, all scans were executed under glucose clamping.

This study did not evaluate regional MPR. Although MPR in this patient group can be expected to show regional differences, global MPR was found to be an important prognostic indicator. This finding may reflect global and not just regional coronary vascular failure. In comparison, dilated cardiomyopathy patients with a left bundle branch block have a lower global perfusion than do patients with no left bundle branch block, despite a heterogeneous perfusion pattern (26).

Finally, one could question what the clinical significance of MPR measurements in these patients is. Most of these patients were not amenable to coronary intervention. We believe that this may be quite relevant because the low MPR may argue in favor of a coronary intervention and of optimally treating patients with a tailored approach to improve endothelial and vascular function.

\section{CONCLUSION}

MPR assessed with PET is an important predictor of cardiac death in patients with IHD not amenable to surgical or percutaneous revascularization. Therefore, therapeutic strategies to improve MPR are of the highest importance always, not just when symptoms are worsening.

\section{REFERENCES}

1. Ross R. Atherosclerosis: an inflammatory disease. N Engl J Med. 1999;340:115126.

2. Galasko GI, Lahiri A. The non-invasive assessment of hibernating myocardium in ischaemic cardiomyopathy: a myriad of techniques. Eur J Heart Fail. 2003; 5:217-227.

3. Tillisch J, Brunken R, Marshall R, et al. Reversibility of cardiac wall-motion abnormalities predicted by positron tomography. N Engl J Med. 1986;314:884-888.

4. Tamaki N, Yonekura Y, Yamashita K, et al. Positron emission tomography using fluorine-18 deoxyglucose in evaluation of coronary artery bypass grafting. Am J Cardiol. 1989;64:860-865.

5. Beanlands RS, Ruddy TD, deKemp RA, et al. Positron emission tomography and recovery following revascularization (PARR-1): the importance of scar and the development of a prediction rule for the degree of recovery of left ventricular function. J Am Coll Cardiol. 2002;40:1735-1743.
6. Hutchins GD, Schwaiger M, Rosenspire KC, Krivokapich J, Schelbert H, Kuhl DE. Noninvasive quantification of regional blood flow in the human heart using N-13 ammonia and dynamic positron emission tomographic imaging. J Am Coll Cardiol. 1990;15:1032-1042.

7. Schindler TH, Nitzsche EU, Schelbert HR, et al. Positron emission tomographymeasured abnormal responses of myocardial blood flow to sympathetic stimulation are associated with the risk of developing cardiovascular events. J Am Coll Cardiol. 2005;45:1505-1512.

8. Cecchi F, Olivotto I, Gistri R, Lorenzoni R, Chiriatti G, Camici PG. Coronary microvascular dysfunction and prognosis in hypertrophic cardiomyopathy. N Engl J Med. 2003;349:1027-1035.

9. Camici PG, Crea F. Coronary microvascular dysfunction. N Engl J Med. 2007; 356:830-840.

10. Neglia D, Michelassi C, Trivieri MG, et al. Prognostic role of myocardial blood flow impairment in idiopathic left ventricular dysfunction. Circulation. 2002; 105:186-193.

11. van den Heuvel AF, van Veldhuisen DJ, van der Wall EE, et al. Regional myocardial blood flow reserve impairment and metabolic changes suggesting myocardial ischemia in patients with idiopathic dilated cardiomyopathy. $\mathrm{J} \mathrm{Am}$ Coll Cardiol. 2000;35:19-28.

12. Blanksma PK, Willemsen ATM, Meeder JG, et al. Quantitative myocardial mapping of perfusion and metabolism using parametric polar map displays in cardiac PET. J Nucl Med. 1995;36:153-158.

13. Knuuti MJ, Yki-Jarvinen H, Voipio-Pulkki LM, et al. Enhancement of myocardial [fluorine-18]fluorodeoxyglucose uptake by a nicotinic acid derivative. J Nucl Med. 1994;35:989-998.

14. Knuuti MJ, Nuutila P, Ruotsalainen U, et al. Euglycemic hyperinsulinemic clamp and oral glucose load in stimulating myocardial glucose utilization during positron emission tomography. J Nucl Med. 1992;33:1255-1262.

15. Gambhir SS, Schwaiger M, Huang SC, et al. Simple noninvasive quantification method for measuring myocardial glucose-utilization in humans employing positron emission tomography and F-18 deoxyglucose. J Nucl Med. 1989;30: 359-366.

16. Desideri A, Cortigiani L, Christen AI, et al. The extent of perfusion-F18fluorodeoxyglucose positron emission tomography mismatch determines mortality in medically treated patients with chronic ischemic left ventricular dysfunction. J Am Coll Cardiol. 2005;46:1264-1269.

17. Sorrentino AR, Acampa W, Petretta M, Mainolfi C, Salvatore M, Cuocolo A. Comparison of the prognostic value of SPECT after nitrate administration and metabolic imaging by PET in patients with ischaemic left ventricular dysfunction. Eur J Nucl Med Mol Imaging. 2007;34:558-562.

18. Wiggers H, Nielsen SS, Holdgaard P, et al. Adaptation of nonrevascularized human hibernating and chronically stunned myocardium to long-term chronic myocardial ischemia. Am J Cardiol. 2006;98:1574-1580.

19. Hachamovitch R, Berman DS, Shaw LJ, et al. Incremental prognostic value of myocardial perfusion single photon emission computed tomography for the prediction of cardiac death: differential stratification for risk of cardiac death and myocardial infarction. Circulation. 1998;97:535-543.

20. Tarakji KG, Brunken R, McCarthy PM, et al. Myocardial viability testing and the effect of early intervention in patients with advanced left ventricular systolic dysfunction. Circulation. 2006;113:230-237.

21. Kaufmann PA, Gnecchi-Ruscone T, di Terlizzi M, Schafers KP, Luscher TF, Camici PG. Coronary heart disease in smokers: vitamin $\mathrm{C}$ restores coronary microcirculatory function. Circulation. 2000;102:1233-1238.

22. Dayanikli F, Grambow D, Muzik O, Mosca L, Rubenfire M, Schwaiger M. Early detection of abnormal coronary flow reserve in asymptomatic men at high risk for coronary artery disease using positron emission tomography. Circulation. 1994;90:808-817.

23. Nitenberg A, Valensi P, Sachs R, Dali M, Aptecar E, Attali JR. Impairment of coronary vascular reserve and ACh-induced coronary vasodilation in diabetic patients with angiographically normal coronary arteries and normal left ventricular systolic function. Diabetes. 1993;42:1017-1025.

24. Epstein SE, Cannon RO III. Site of increased resistance to coronary flow in patients with angina pectoris and normal epicardial coronary arteries. J Am Coll Cardiol. 1986;8:459-461.

25. de Boer RA, Pinto YM, van Veldhuisen DJ. The imbalance between oxygen demand and supply as a potential mechanism in the pathophysiology of heart failure: the role of microvascular growth and abnormalities. Microcirculation. 2003;10:113-126.

26. Lindner O, Vogt J, Baller D, et al. Global and regional myocardial oxygen consumption and blood flow in severe cardiomyopathy with left bundle branch block. Eur J Heart Fail. 2005;7:225-230. 